\title{
Hair Growth-Promoting Effects of Lavender Oil in C57BL/6 Mice
}

\author{
Boo Hyeong Lee', Jae Soon Lee ${ }^{2}$ and Young Chul Kim ${ }^{3}$ \\ ${ }^{1}$ Department of Beauty Art, Changwon Moonsung University, Changwon, Korea \\ ${ }^{2}$ Department of Beauty Design, Gyongbuk Science College, Chilgok, Korea \\ ${ }^{3}$ Department of Public Health, Keimyung University, Daegu, Korea
}

(Received November 24, 2015; Revised December 15, 2015; Accepted December 22, 2015)

\begin{abstract}
The purpose of this study was to determine the hair growth effects of lavender oil (LO) in female C57BL/6 mice. The experimental animals were divided into a normal group ( $\mathrm{N}$ : saline), a vehicle control group (VC: jojoba oil), a positive control group (PC: 3\% minoxidil), experimental group 1 (E1: 3\% LO), and experimental group 2 (E2: $5 \%$ LO). Test compound solutions were topically applied to the backs of the mice $(100 \mu \mathrm{L}$ per application), once per day, 5 times a week, for 4 weeks. The changes in hair follicle number, dermal thickness, and hair follicle depth were observed in skin tissues stained with hematoxylin and eosin, and the number of mast cells was measured in the dermal and hypodermal layers stained with toluidine blue. PC, E1, and E2 groups showed a significantly increased number of hair follicles, deepened hair follicle depth, and thickened dermal layer, along with a significantly decreased number of mast cells compared to the $\mathrm{N}$ group. These results indicated that LO has a marked hair growth-promoting effect, as observed morphologically and histologically. There was no significant difference in the weight of the thymus among the groups. However, both absolute and relative weights of the spleen were significantly higher in the PC group than in the N, VC, E1, or E2 group at week 4. Thus, LO could be practically applied as a hair growth-promoting agent.
\end{abstract}

Key words: C57BL/6 mice, Dermal thickness, Hair growth, Hair follicle, Lavender oil

\section{INTRODUCTION}

Hair serves as a cushion against external shocks, as a means to keep the head warm, and a shield to protect the scalp from ultraviolet rays. It is also responsible for excreting harmful heavy metals, such as arsenic, mercury, zinc, etc., removing them from the body, and plays an important role in beauty, as the means of expressing the appearance of a person (1). Although generally thought to be linked to aging, hair loss has many direct and indirect causes, such as increased stress, increased social activities in women, nutritional imbalances due to westernized diets, in addition to genetic factors (2). In cases of severe hair loss, there can be negative psychological consequences, such as lowered selfesteem and anger, which can then lead to depression, social

Correspondence to: Young Chul Kim, Department of Public Health, Keimyung University, 1095 Dalgubeol-daero, Daegu 42601, Korea E-mail: yckim@kmu.ac.kr

This is an Open-Access article distributed under the terms of the Creative Commons Attribution Non-Commercial License (http:// creativecommons.org/licenses/by-nc/3.0) which permits unrestricted non-commercial use, distribution, and reproduction in any medium, provided the original work is properly cited. withdrawal, and inappropriate personal relations, etc. (3). Therefore, since hair loss is no longer regarded as the simple elimination of hair, but is linked to social and psychological pain, understanding of the importance of proper management is gaining momentum.

Hair follicles (HFs) are depressions in the scalp in which hair is created through the continuous proliferation of matrix cells, in a growth cycle that includes various phases (4). A hair cycle in a normal scalp is classified into the anagen phase, which lasts 3 6 years and involves active growth; the catagen phase, which lasts 3 4 weeks and involves halting and contracting of growth activity; and the telogen phase, which lasts 3 4 months and is the period when the hair eventually falls out and is replaced by a new hair. The hair cycle is repeated 10 20 times during a lifetime $(5,6)$.

Minoxidil (MXD), a pyrimidine derivative (2,4-diamino6-piperidino-pyrimidine-3-oxide) is an adenosine triphosphate (ATP)-sensitive potassium channel (KATP channel) opener, and is the most commonly used drug for the treatment of androgenetic alopecia (7). Topical MXD shortens the telogen phase by inducing resting HFs into the anagen phase, prolongs the anagen growth phase, and increases the volume of HFs. 
Lavender oil (LO) constituents include linalyl acetate, linalool, and geraniol. It is known to be effective for the recovery of fatigue, the relaxation of stress, the growth of cells, the reproduction of skin, and alopecia areata (8). Among these constituents, linalyl acetate ester is effective for the relaxation of nerves, and as a tincture, linalool has antifungal effects (9). Aroma therapy is one of the alternative medicines that are currently gaining much attention.

This study investigated the practicality of using LO as a hair growth stimulant, as compared to treatment with $3 \%$ MXD, which has been approved by the US-FDA, and to provide basis for the development of hair loss treatments. In order to do this, the hair-growth promoting effect of LO was determined through morphological and histological observation, by percutaneous application of LO to the back skin of C57BL/6 mice. In addition, the safety of this treatment was assessed through the observation and measurement of organ weights.

\section{MATERIALS AND METHODS}

Materials. LO was purchased from Sanoflore Company (France) and jojoba oil was purchased from Bio \& Bio (Korea). The chemical composition of LO and jojoba oil is listed in Table 1. The 3\% MXD was obtained from Hyundai Pharmacia (Korea). In order to prepare the 3\% and 5\% solutions, LO was mixed with jojoba oil.

Experimental animals. Five-week-old female C57BL/ 6 mice were purchased from Dae Han Biolink Co (Korea). The animals were housed and allowed to adapt to the laboratory environment for 1 week, after which the 6-week-old mice were divided into 5 groups of 18 mice each: a saline treatment group, as the normal group $(\mathrm{N})$; a jojoba oil treatment group, as the vehicle control group (VC); a 3\% MXD

Table 1. Composition of lavender oil and jojoba oil

\begin{tabular}{lrlr}
\hline \hline Peppermint oil & \multicolumn{1}{c}{$\%$} & Jojoba oil & \multicolumn{1}{c}{$\%$} \\
\hline Linalyl acetate & 38.8 & Docosenyl elcosenoate & 41.5 \\
Linalool & 31.7 & Elcosenyl elcosenoate & 27.7 \\
Trans- $\beta$-3-ocimene & 4.9 & Elcosenyl docosenoate & 10.3 \\
Cis- $\beta$-ocimene & 3.0 & Tetracosanyl elcosanate & 6.3 \\
Lavandulyl acetate & 2.3 & Elcosenyl oleate & 5.4 \\
Terpinene-4-ol & 1.3 & Docosenyl stearate & 3.2 \\
a-Terpineol & 0.6 & Other esters & 5.6 \\
Camphre & 0.6 & & \\
1,8-Cineole & 0.6 & & \\
Lavandulol & 0.4 & & \\
$\beta$-Phellandrene & 0.2 & & \\
Octanone 3 & 0.2 & & \\
Limonene & 0.2 & & \\
The others & 15.2 & & \\
\hline \multicolumn{1}{c}{ Total } & 100.0 & & Total \\
\hline
\end{tabular}

treatment group, as the positive control group (PC); a 3\% lavender oil (LO) treatment group, as experiment group 1 (E1), and a 5\% lavender oil (LO) treatment group, as experiment group 2 (E2). To assess the effects of hair growth, the back of each mouse was shaved 1 day before the beginning of the experiment, using an electric clipper for animals, and $100 \mu \mathrm{L}$ of test samples was applied topically by using micro pipettes, once a day, 5 times a week, for 4 weeks. The temperature of the animal laboratory environment was maintained at $22 \pm 1{ }^{\circ} \mathrm{C}$, with a relative humidity of $50 \pm 5 \%$, and an alternating 12-hr light/dark cycle; animals were provided with food and water ad libitum. Both the animal care and the protocol for this study were in accordance with IACUC (Institutional Animal Care and Use Committee) and OECD guidelines (Approval No. KMU IACUC 2011012).

Six experimental animals were euthanized at weeks 1, 3 , and 4, using ether anesthesia, after which the organs were immediately extracted and washed in physiological saline, dried off with filter paper; the spleen and thymus were weighed. A part of the extracted skin tissue was fixed in $10 \%$ formalin solution and used for histological observation.

Hair growth observation. At weeks 1, 3, and 4, the backs of the mice were photographed with a digital camera under ether anesthesia, to evaluate the darkening of skin color. The hair growth effect in each group was rated on a score ranging from 0 to $100 \%$.

Histological analysis. Mice were euthanized with diethyl ether and skin tissue extracted. The number of mice sacrificed at weeks 1, 3, and 4 was 6 each, and the dermal skin samples were fixed in $10 \%$ buffered formalin for $24 \mathrm{hr}$, followed by embedding in paraffin wax, using standard techniques. General histology was visualized using hematoxylineosin (H\&E) staining. We observed the number, elongation, and depth of HFs by fluorescent microscopy (Axio imager, Carl Zeiss, Germany). The dermal thickness and follicle depth were measured using the scale bar tool of the fluorescent microscope. The number of mast cells (MCs) in the dermal and subcutaneous layers was counted after toluidine blue staining.

Statistical analysis. A one-way ANOVA analysis was conducted using SPSS 17.0 for Windows (SPSS Inc., USA) statistics program; post hoc comparison was used to test the differences between the groups, using Duncan's multiple range test. Statistical significance was set at $p<0.05$.

\section{RESULTS}

Gross observation. In morphological observation (Fig. $1)$, hair growth area was determined as \% after photograph- 

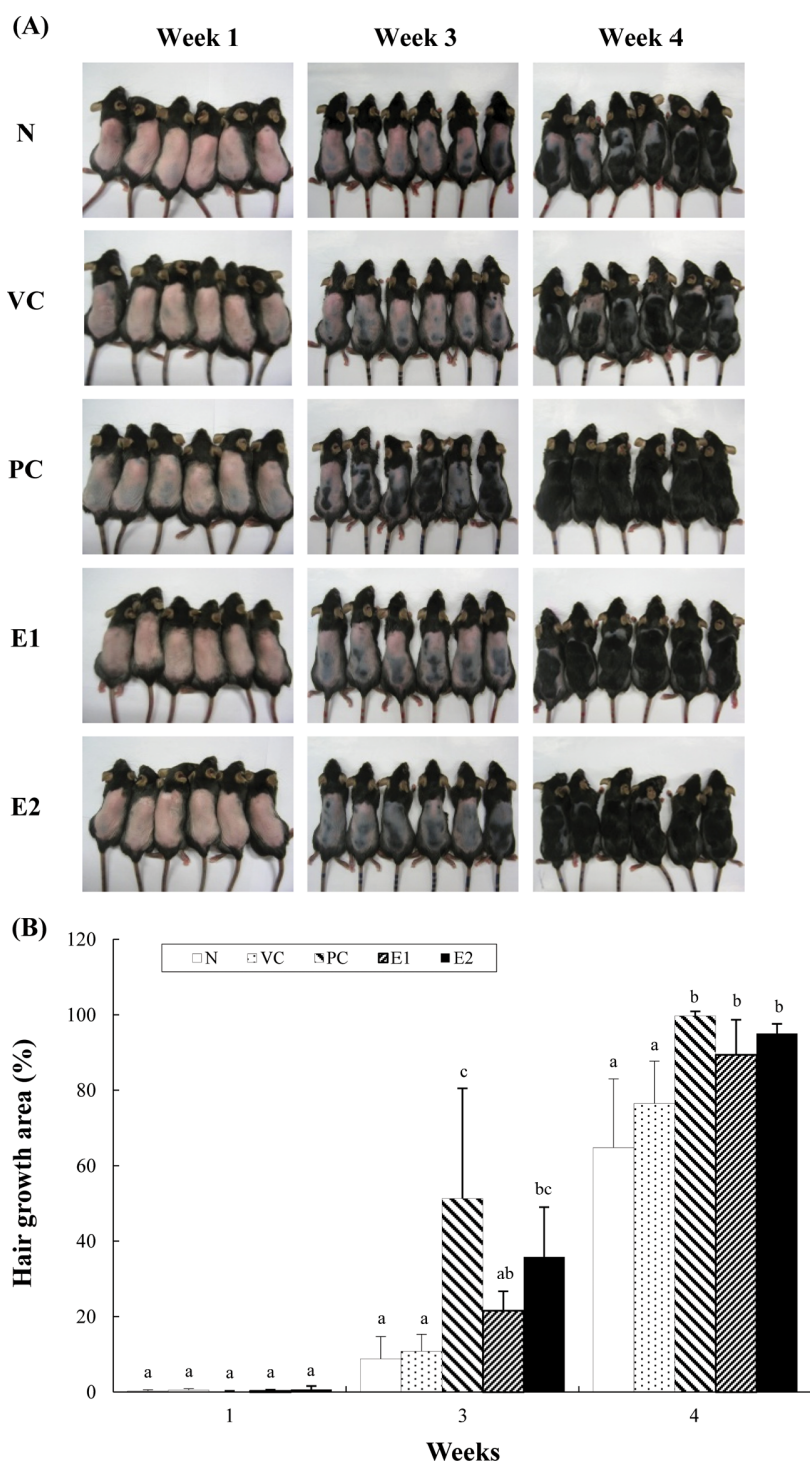

Fig. 1. Hair growth effects in an alopecia model of C57BL/6 mice to which test compounds were applied topically for 4 weeks. N: saline, VC: jojoba oil, PC: 3\% minoxidil, E1: 3\% lavender oil, E2: 5\% lavender oil. (A) Photographs of the back skin. From week 3, PC, E1, and E2 groups showed notable darkening of skin color, and over time they showed a markedly greater hair growth than the $\mathrm{N}$ group. (B) Hair growth area of back skin. Each value represents the mean \pm SD of 6 mice. ${ }^{a, b, c}$ Values with different superscripts indicate significant differences $(p<0.05)$ for each given week, by ANOVA and Duncan's multiple range tests.

ing hair growth state on the backs of mice and observation was conducted 3 times in total, i.e., at weeks 1, 3 and 4. At the $1^{\text {st }}$ week of the experiment, less than $1 \%$ of hair growth was seen in all 5 groups. At the $3^{\text {rd }}$ week, the PC, E1, and E2 groups showed $51 \%, 22 \%$, and $36 \%$ of hair growth, respectively, and at the $4^{\text {th }}$ week, PC, E1, and E2 groups showed $99.8 \%, 90 \%$, and $95 \%$ of hair growth, respectively.
Optical microscopic observations with H \& E staining. The observation of HF number, HF depth, and dermal thickness of the back skin using an optical microscope are shown in Fig. 2. During the experimental period, the HF number in the PC, E1 and E2 groups increased; HF depth was deeper and dermal thickness was thicker than in the $\mathrm{N}$ group. The HF number, HF depth, and dermal thickness were significantly $(p<0.05)$ increased in the PC, E1, and $\mathrm{E} 2$ groups than in the $\mathrm{N}$ group at the $3^{\text {rd }}$ or $4^{\text {th }}$ week of the experiment.

Change in the number of mast cells. The number of MCs was measured in the dermal and subcutaneous layers by toluidine blue staining, which were visually checked at a $200 \times$ magnification, using an optical microscope (Fig. 3). At the $1^{\text {st }}$ week, the number of MCs was significantly $(p<$ 0.05 ) higher in the $\mathrm{N}$ and $\mathrm{VC}$ groups than in the PC, E1, and E2 groups. As time passed, these values slightly decreased in all groups. At the $4^{\text {th }}$ week, the number of MCs was in the order of $\mathrm{N}>\mathrm{VC}>\mathrm{E} 1>\mathrm{E} 2>$ PC groups.

Organ weight. The weight of the spleen and thymus is shown in Fig. 4. At the $4^{\text {th }}$ week of the experiment, the absolute weight of the spleen in the PC group was approximately $14 \%(p<0.05)$ higher than that in the $\mathrm{N}$ group, but those in the E1 and E2 groups were not significantly different from that in the $\mathrm{N}$ group. There was no significant difference in the absolute weight of the thymus between groups.

At the $4^{\text {th }}$ week of the experiment, the relative weight (g/ $100 \mathrm{~g}$ body weight) of the spleen in the PC group was approximately $9 \%(p<0.05)$ higher than in the $\mathrm{N}$ group, but those in the E1 and E2 groups were not significantly different from that in the $\mathrm{N}$ group. There was no significant difference in the relative weight of the thymus between groups.

\section{DISCUSSION}

C57BL/6 mice are widely used in hair physiology studies because they have the advantage that all HFs enter the growth stage after birth, with hair beginning to grow, and with spontaneous hair loss when all HFs transfer to the pause stage at around 6 weeks. The growth stage can be determined by skin color, because melanocytes exist only in HFs and melanin synthesis matches the cycle of hair growth well $(10,11)$. In this study, 6 week-old female C57BL/6 mice were used. When the hair on their back was epilated or cut, the back skin color was pink; as the experiment progressed, the surface of the skin changed from pink to black through gray, corresponding with the change in the hair cycle from the pause stage to the growth stage. In this study, hair growth was not observed morphologically after topical application of LO to the back skin for 1 week. Hair growth 

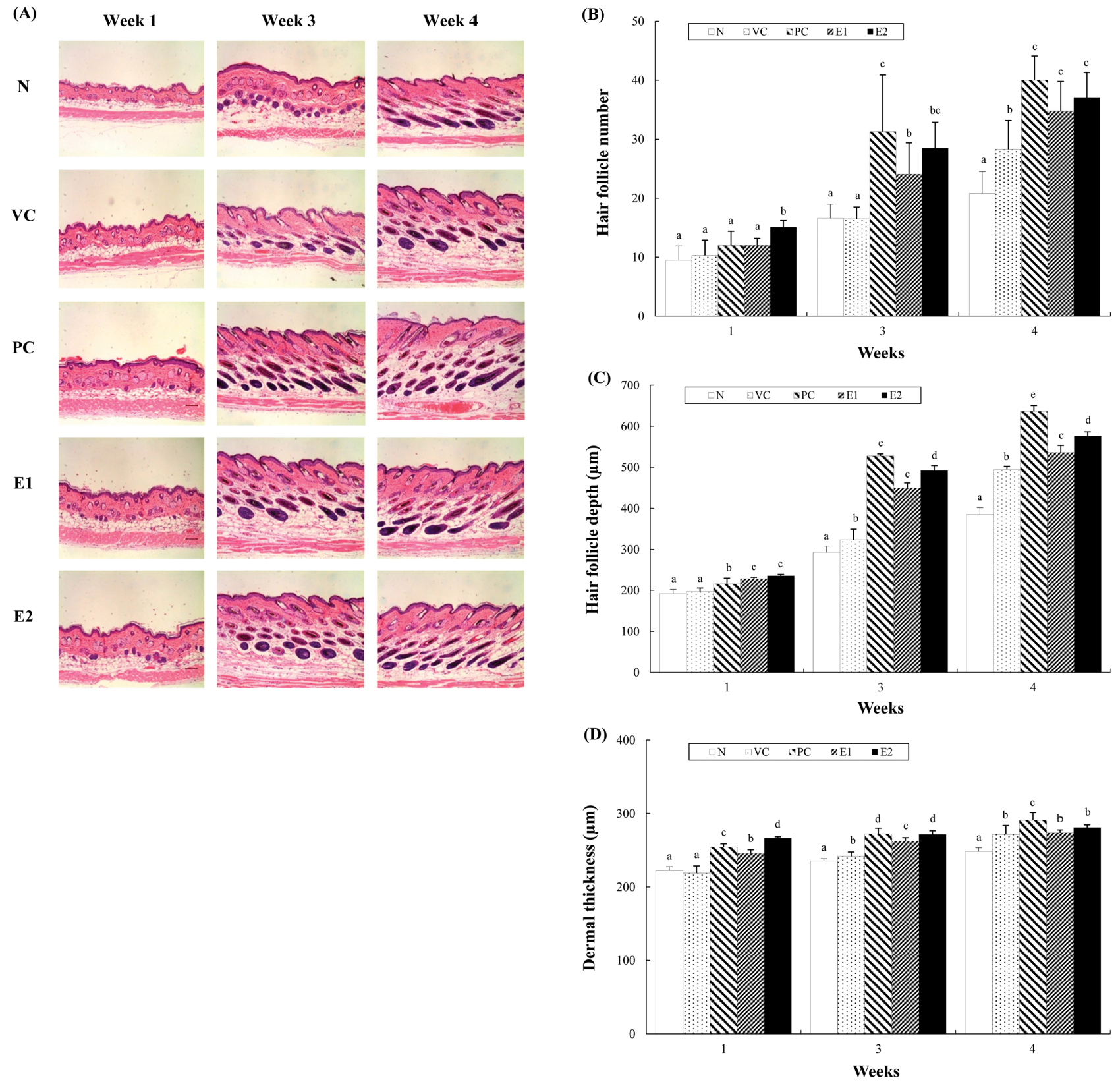

Fig. 2. Histological observation of hair follicles and dermal thickness in the back skin of C57BL/6 mice to which test compounds were applied topically for 4 weeks. N: saline, VC: jojoba oil, PC: 3\% minoxidil, E1: 3\% lavender oil, E2: 5\% lavender oil. (A) Hair follicles. Hematoxylin and eosin staining, $\times 100$. (B) Hair follicle number. (C) Hair follicle depth. (D) Dermal thickness. As the experiment progressed, the hair follicle number and depth in the PC, E1, and E2 groups increased markedly as compared to the N and VC groups. The dermal layer was also observed to be thicker in the PC, E1, and E2 groups. ${ }^{a, b, c, d, e}$ Values with different superscripts indicate significant differences $(p<0.05)$ for each given week, by ANOVA and Duncan's multiple range tests.

was clearly visible on the back skin in the order of $\mathrm{PC}>$ $\mathrm{E} 2>\mathrm{E} 1>\mathrm{VC}>\mathrm{N}$ groups after application of the test compounds for 3 weeks. At week 4, a marked hair growth-promoting effect was observed in the PC, E1, and E2 groups, as compared to the $\mathrm{N}$ and $\mathrm{VC}$ groups.

To perform a histological assessment of HFs, skin tissue was cut from the back and stained with H\&E, and was observed under an optical microscope. At week 4 of the experiment, there was significant increase in $\mathrm{HF}$ number, $\mathrm{HF}$ depth, and dermal thickness in the PC, E1, and E2 groups, as compared to the $\mathrm{N}$ and VC groups, which supported the morphological observation of the hair growthpromoting effect of LO. A change in the form of the HF is closely related to the hair cycle. HFs become longer during 
(A)
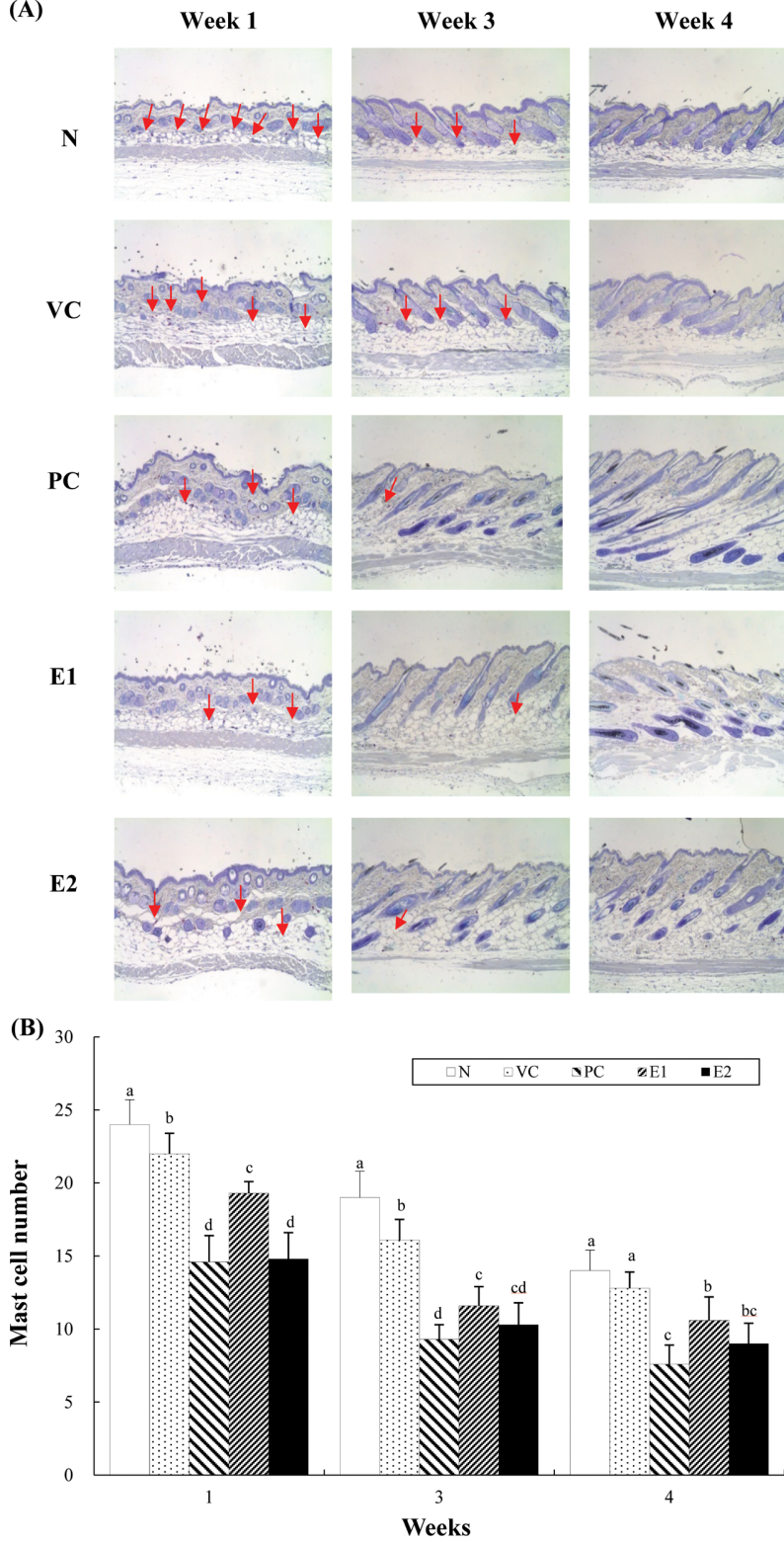

Fig. 3. Histological observation of mast cells in the back skin of C57BL/6 mice to which test compounds were applied topically for 4 weeks. N: saline, VC: jojoba oil, PC: $3 \%$ minoxidil, E1: 3\% lavender oil, E2: 5\% lavender oil. (A) Mast cells. Toluidine blue staining, $\times 200$. In the PC, E1, and E2 groups, the number of mast cells (arrows marks) gradually decreased as compared to the $\mathrm{N}$ group. (B) Mast cell number. Each value represents the mean \pm SD of 6 mice. ${ }^{a, b, c, d}$ Values with different superscripts at a given week are significantly $(p<0.05)$ different by ANOVA and Duncan's multiple range tests.

the growth stage (steps I-VI), and become shorter during the degeneration stage (steps I-VIII). They are longest at step VI of the growth stage and maintain the same length at steps I-II of the degeneration stage, after which it decreases, and is shortest at the pause stage (11). Continuous growth
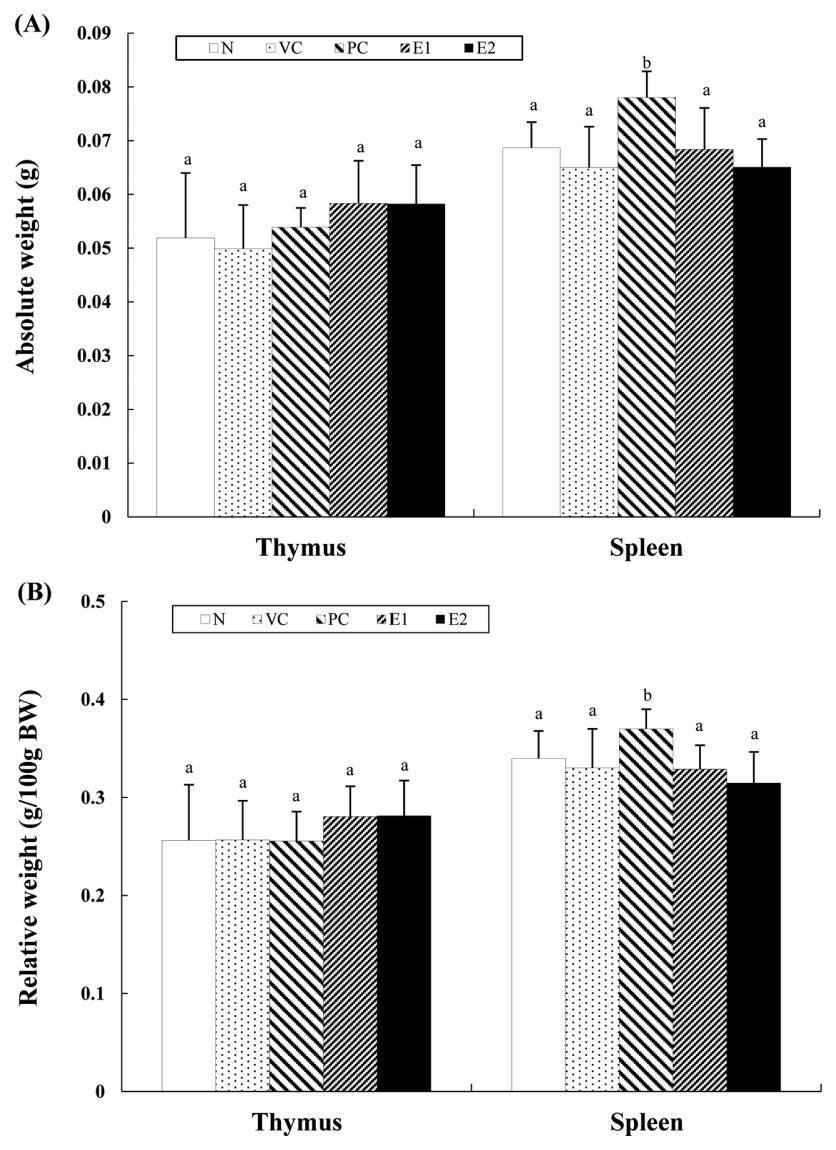

Fig. 4. Comparison in the organ weights of the thymus and spleen of C57BL/6 mice to which test compounds were applied topically for 4 weeks. N: saline, VC: jojoba oil, PC: $3 \%$ minoxidil, E1: 3\% lavender oil, E2: 5\% lavender oil. (A) Absolute weight. (B) Relative weight. Each value represents the mean \pm SD of 6 mice. ${ }^{a, b}$ Values with different superscripts indicate significant differences $(p<0.05)$, by ANOVA and Duncan's multiple range tests.

of HFs during the growth stage and degeneration of HFs during the degeneration stage are also related to changes in the cycle of skin thickness. From the pause stage to step II of the growth stage, HFs are limited to the dermal layer, and the epidermal layer becomes thick; from step III of the growth stage, as HFs are depressed to the subcutaneous layer, the epidermal layer becomes thin and the dermal layer and subcutaneous layer become thicken (12).

MCs are distributed around HFs, and the murine hair cycle is widely associated with the degranulation of mast cells. Degranulated perifollicular MCs decrease markedly shortly after the induction of hair growth (anagen) and significantly increase during late anagen VI, immediately before the onset of catagen. Catagen development is detained by inhibition of MC degranulation $(13,14)$. Stressors induce the production of corticotropin-releasing hormone in HFs, which increases the degranulation of MCs. In addition, the corticotropin-releasing hormone causes an increase in the 
number of MCs, which may induce catagen by chemical mediators from MCs (15). The number of MCs was determined by toluidine blue staining. In this study, at weeks 1 , 3 , and 4, the number of MCs in the PC, E1, and E2 groups was significantly $(p<0.05)$ lower than in the $\mathrm{N}$ and $\mathrm{V}$ groups. Therefore, LO maintains the anagen phase and delays the transition to the catagen phase. Sandalwood oil also decreases the number of MCs, along with promoting hair growth in $\mathrm{C} 57 \mathrm{BL} / 6$ mice (16).

To verify the basic safety of LO, the organ weights of spleen and thymus was measured. At week 4 of the experiment, the absolute weight and relative weight of the spleen were significantly $(p<0.05)$ higher in the PC group than in the N, VC, E1, and E2 groups. On the other hand, the absolute weight and relative weight of the thymus were not significantly different among the groups.

Taken together, the results of this study show the marked hair growth-promoting effect of LO, as evidenced by morphological and histological observations.

\section{REFERENCES}

1. Hadshiew, I.M., Foitzik, K., Arck, P.C. and Paus, R. (2004) Burden of hair loss: stress and the underestimated psychosocial impact of telogen effluvium and androgenetic alopecia. $J$. Invest. Dermatol., 123, 455-457.

2. Rho, S.S., Park, S.J., Hwang, S.L., Lee, M.H., Kim, C.D., Lee, I.H., Chang, S.Y. and Rang, M.J. (2005) The hair growth promoting effect of Asiasari radix extract and its molecular regulation. J. Dermatol. Sci., 38, 89-97.

3. Cash, T.F., Price, V.H. and Savin, R.C. (1993) Psychological effects of androgenetic alopecia on women: comparisons with balding men and with female control subjects. J. Am. Acad. Dematol., 29, 568-575.

4. Alonso, L. and Fuchs, E. (2006) The hair cycle. J. Cell Sci., 119, 391-393.

5. Paus, R., Christoph, T. and Müller-Röver, S. (1999) Immunology of the hair follicle: a short journey into terra incognita. $J$.
Investig. Dermatol. Symp. Proc., 4, 226-234.

6. Van Neste, D. and Tobin, D.J. (2004) Hair cycle and hair pigmentation: dynamic interactions and changes associated with aging. Micron, 35, 193-200.

7. Messenger, A.G. and Rundegren, J. (2004) Minoxidil: mechanisms of action on hair growth. Br. J. Dermatol., 150, 186194.

8. Guin, J.D. (1981) Complications of topical hydrocortisone. $J$. Am. Acad. Dermatol., 4, 417-422.

9. Bouseta, A., Scheirman, V. and Collin, S. (1996) Flavor and free amino acid composition of lavender and eucalyptus honeys. J. Food Sci., 61, 683-687.

10. Slominski, A. and Paus, R. (1993) Melanogenesis is coupled to murine anagen: toward new concepts for the role of melanocytes and the regulation of melanogenesis in hair growth. $J$. Invest. Dermatol., 101, 90S-97S.

11. Müller-Röver, S., Handjiski, B., van der Veen, C., Eichmüller, S., Foitzik, K., Mckay, I.A., Stenn, K.S. and Paus, R. (2001) A comprehensive guide for the accurate classification of murine hair follicles in distinct hair cycle stages. J. Invest. Dermatol., 117, 3-15.

12. Sun, L.Q., Lee, D.W., Zhang, Q., Xiao, W., Raabe, E.H., Meeker, A., Miao, D., Huso, D.L. and Arceci, R.J. (2004) Growth retardation and premature aging phenotypes in mice with disruption of the SNF2-like gene, PASG. Genes Dev., 18, 1035-1046.

13. Paus, R. and Link, R.E. (1988) The psoriatic epidermal lesion and angen hair growth may share the same "switch-on" mechanism. Yale J. Biol. Med., 61, 467-476.

14. Paus, R., Maurer, M., Slominski, A. and Czametzki, B.M. (1994) Mast cell involvement in murine hair growth. Dev. Biol., 163, 230-240.

15. Ito, N., Sugawara, K., Bodó, E., Takigawa, M., van Beek, N., Ito, T. and Paus, R. (2010) Corticotropin-releasing hormone stimulates the in situ generation of mast cells from precursors in the human hair follicle mesenchyme. J. Invest. Dermatol., 130, 995-1004.

16. Lee, B.S. (2014) Effects of sandalwood oil on aspects of hair growth in a C57BL/6 mouse model of alopecia. J. Invest. Cosmetol., 10, 279-287. 